\title{
Role of Host Sensitivity to Ptr ToxA in Development of Tan Spot of Wheat
}

\author{
T. L. Friesen, S. Ali, S. Kianian, L. J. Francl, and J. B. Rasmussen
}

First, second, fourth, and fifth authors: Department of Plant Pathology; and third author: Department of Plant Sciences, North Dakota State University, Fargo 58105.

Current address of T. L. Friesen: Northern Crops Science Laboratory, U.S. Department of Agriculture, Fargo, ND 58105. A portion of this research was completed by the senior author at this facility.

Accepted for publication 24 October 2002.

\section{ABSTRACT}

Friesen, T. L., Ali, S., Kianian, S., Francl, L. J., and Rasmussen, J. B. 2003. Role of host sensitivity to Ptr ToxA in development of tan spot of wheat. Phytopathology 93:397-401.

Pyrenophora tritici-repentis race 2 produces Ptr ToxA, a host-selective toxin previously described as a pathogenicity factor for tan spot on wheat. The objective of this research was to evaluate the role of host sensitivity to toxin, conditioned by a single dominant gene on chromosome $5 \mathrm{BL}$, in the disease development by race 2 . An $\mathrm{F}_{2}$-derived $\mathrm{F}_{6}$ recombinant inbred population of 108 wheat lines, produced from crosses of toxin-sensitive, disease-susceptible cv. Kulm with the toxin-insensitive, disease-resistant cv. Erik segregated 1:1 for toxin reaction. However, the population was skewed toward resistance to race 2 of the fungus. Toxin reaction accounted for $24.4 \%$ of the genetic variance for disease. Heritability estimates suggested the presence of four to five genes that influence disease reaction in the population. Toxin-insensitive mutants, previously derived Kulm, were susceptible to race 2, although disease developed more slowly on the mutants than it did on the wildtype Kulm. The data indicate that sensitivity to Ptr ToxA influences disease severity in some host genotypes without defining susceptibility.
Tan spot, caused by the fungus Pyrenophora tritici-repentis ((Died.) Drechs.), is an important foliar disease of wheat (Triticum aestivum L.) in many production areas of the world, including the central and northern Great Plains where most U.S. production occurs. The fungus, which overwinters as pseudothecia in stubble, emerged as a threat to wheat production in the 1970s with the advent of reduced tillage practices. Disease is often found in commercial fields when susceptible cultivars are grown and the environment supports infection. Races 1 and 2 of the fungus, both prevalent in North American surveys $(1,13)$, produce the necrosisinducing host-selective toxin (HST) designated Ptr ToxA (2).

The best approach for controlling tan spot is through genetic resistance in accepted cultivars. A long-range objective of our research is to understand the genetic bases of pathogenicity and resistance in this pathosystem, including the role of host sensitivity to Ptr ToxA in disease development. Lamari and Bernier $(11,12)$ conducted the first studies that related the genetics of host sensitivity to Ptr ToxA and susceptibility to the fungus. Their data indicated that both characteristics were controlled by the same dominant gene that subsequently was mapped to host chromosome 5BL (7). The wheat cv. Erik, an older cultivar that is not widely grown, was reported to possess a single recessive gene that conditioned insensitivity to the toxin and resistance to the fungus (12). Erik has since been widely used as a resistant control for experiments with tan spot and Ptr ToxA $(8,9)$ and has been suggested as a source of resistance to tan spot in future cultivar development (17).

A perfect correlation between toxin and disease reaction in segregating populations of the host led to the conclusion that Ptr ToxA is a pathogenicity factor for tan spot (11). Molecular studies of toxin production in the fungus also concluded that Ptr ToxA

Corresponding author: J. B. Rasmussen

E-mail address: jack.rasmussen@ndsu.nodak.edu

Publication no. P-2003-0217-01R

(C) 2003 The American Phytopathological Society was necessary and sufficient for disease development (3). Those data collectively would suggest that Ptr ToxA has a role in disease similar to some other HSTs such as victorin, produced by Cochliobolus victoriae Nelson and specific for oats (Avena sativa L.) with the genetically-dominant $V b$ gene, and HC toxin, produced by $C$. carbonum Nelson race 1 and specific for certain maize (Zea mays L.) inbreds and hybrids. In each of these models, a single host locus controls reactions to the fungus and the corresponding HST produced, and insensitivity to the toxin invariably means resistance to the fungus (18).

However, other data curiously suggest that host reaction to $\mathrm{Ptr}$ ToxA may not by itself determine the host's reaction to $P$. triticirepentis. For example, resistance to tan spot has been described as quantitative and involving additive genes $(5,16)$ and the resistance in Erik as controlled by multiple genes (19). Our group recently isolated three independent wheat mutants insensitive to Ptr ToxA (8). The mutants, derived from the treatment of the toxin-sensitive and tan spot-susceptible cv. Kulm with the chemical mutagen ethylmethane sulfonate (EMS), were susceptible to two race 1 field isolates of the fungus. That work proved that insensitivity to Ptr ToxA is not necessarily equated to resistance to race 1 . This result, though unexpected, was not interpreted to have implications for the role in disease caused by race 2 (8).

Race 1 causes necrotic lesions that usually are surrounded by chlorosis $(n e c+c h l+)$ on wheat $(10,14)$. Our work with the toxininsensitive mutants suggests that race 1 possesses pathogenicity determinants other than Ptr ToxA (8), and it is possible that some of the recently described chlorosis-inducing toxins (4) fulfill this role. By comparison, race 2 produces necrotic lesions only (nec+ chl-). The inability of race 2 to cause chlorosis has contributed to the widespread use of its phenotype (nec+ chl-) in studies that described Ptr ToxA as a pathogenicity determinant $(3,11,12)$. To date, race 2 has not been reported to produce any pathogenicity or virulence factor other than Ptr ToxA.

The objective of the current research was to investigate the role of host sensitivity to Ptr ToxA in disease development by P. tritici- 
repentis race 2 . Two independent lines of experimentation were used. First, toxin and disease reactions were determined for an $\mathrm{F}_{2}$ derived $\mathrm{F}_{6}$ recombinant inbred (RI) population of wheat that was developed from crosses of Kulm (toxin-sensitive and diseasesusceptible) and Erik (toxin-insensitive and disease-resistant). Second, the Ptr ToxA-insensitive mutants were evaluated for their reaction to race 2 .

\section{MATERIALS AND METHODS}

Fungus and toxin. $P$. tritici-repentis race 2 (isolate 86-124), originally obtained from L. Lamari, University of Manitoba, was used for production of conidia for inoculations and for Ptr ToxA. Conidia were produced and adjusted to a concentration of 3,000 per ml (17). Purified Ptr ToxA, obtained as described (20), was provided by S. Meinhardt, Department of Biochemistry, North Dakota State University.

Host material and populations. All experiments made use of the hard red spring wheat cvs. Erik and Kulm. Some experiments used three toxin-insensitive mutants (M37, M103, and M322) derived from EMS treatment of Kulm or homozygous toxin-sensitive or homozygous toxin-insensitive $\mathrm{F}_{3}$ families derived from mutant $\times$ Kulm crosses produced and identified as described (8). Fifty-four RI lines were derived from a cross of Kulm $\times$ Erik and 54 lines from the reciprocal cross. All lines were $\mathrm{F}_{2}$ derived and were produced by single seed descent through the $\mathrm{F}_{6}$ generation in the greenhouse. A single head was bagged prior to anthesis during each generation to ensure self-pollination. Seed of each $F_{6}$ line were increased in the greenhouse to permit replicated experiments with each line.

Toxin bioassay. Wheat seedlings were produced in a growth chamber (12-h photoperiod, $21^{\circ} \mathrm{C}$ ), one seedling per "conetainer" (Stuewe \& Sons, Inc., Corvallis, OR), for 17 to 21 days prior to use in toxin bioassays or inoculations experiments. The second leaf was infiltrated with Ptr ToxA $\left(10 \mu \mathrm{g} \mathrm{m}^{-1}\right)$, after which the seedling was returned to the growth chamber (12-h photoperiod at $21^{\circ} \mathrm{C}$ ). After a 72-h incubation, the infiltrated region was evaluated for the presence or absence of necrosis development (8).

Inoculations and disease evaluation. Ten individuals of each wheat genotype or RI line were divided into two replicates of five

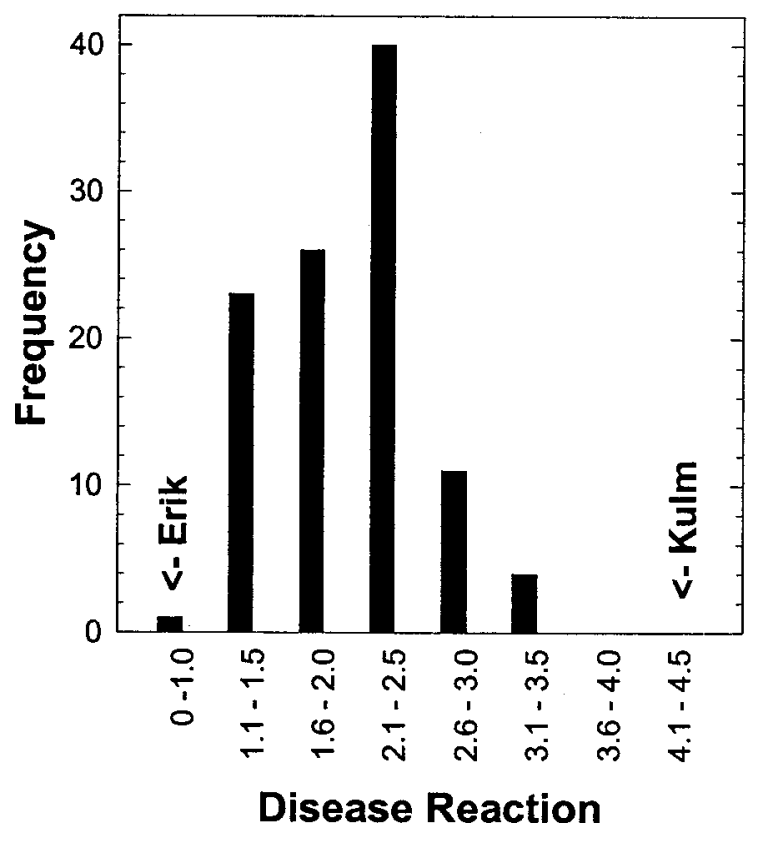

Fig. 1. Frequency distribution of disease severities for 106 recombinant inbred lines. Disease data are average of four experiments scored on a scale of 1 (resistant) to 5 (susceptible) 7 days postinoculation. Average disease reactions for the parents were 1.0 (Erik) and 4.3 (Kulm). seedlings each, then were inoculated as described (17). Disease evaluations were slightly modified from Lamari and Bernier (10). In that system, evaluations are made 6 to 8 days postinoculation on a scale of 1 (dark brown to black spots corresponding to resistance) to 5 (susceptible). A score of 2 (dark spots surrounded by slight necrosis) is considered moderately resistant (MR), a score of 3 is MR/moderately susceptible (MS), and 4 is MS (10). We retained features 1 to 5 of the scale, but added a " 0 " rating to indicate the absence of disease lesions $24 \mathrm{~h}$ after inoculation. This extension of the disease evaluation scale facilitated the development of comparative disease progress curves between the wheat genotypes for some experiments.

Inoculated plants of each RI line were evaluated for disease severity once at 7 days postinoculation. In some experiments involving toxin-insensitive mutants, the same set of plants were evaluated on multiple days between 1 and 8 days postinoculation to construct disease progress curves in the growth chamber. All evaluations of inoculated mutants or progeny of crosses where a mutant was a parent were performed in a randomized, "blind" manner, such that the genotype of each seedling was unknown to the reader and that the individuals within a replicate were not read in sequence.

Statistical analyses. Disease ratings reported for the RI lines are the average of four independent experiments. The proportion of phenotypic variance in the RI population with significant association between toxin and disease reactions was determined by linear regression analysis (15). Formulas from Falconer and Mackay (6) were used for estimates of heritability of resistance and to estimate the number of genes associated with reaction to the fungus in the population. The heritability estimate $\left(H^{2}=\right.$ $\sigma_{\text {genetic }}{ }^{2} /\left[\sigma_{\text {genetic }}{ }^{2}+\sigma_{\text {error }}^{2}\right] /$ reps) was based on analysis of variance to estimate the genetic effect partitioned from the environmental effect. By eliminating the variance due to environment or sampling error, it was possible to more accurately estimate the amount of variance due primarily to the genotype. The formula $n=R^{2}{ }_{\mathrm{T}} / 8 \sigma^{2}$ was used to estimate the number of resistance genes in the population. In this formula, $R_{\mathrm{T}}$ is the difference between the upper and lower selection limits, and $\sigma^{2}$ is the additive variance $\left(\sigma^{2}=\right.$ $\left[\sigma_{\text {line }}^{2}+\sigma_{\text {error }}^{2}\right] /$ number of replicates) in the base population. The

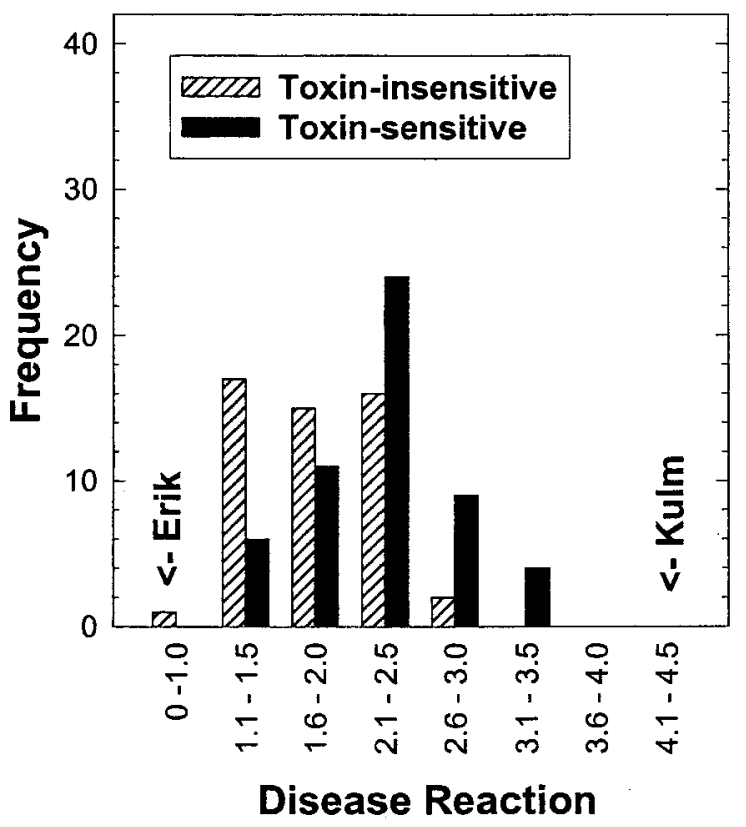

Fig. 2. Distribution of disease reactions for the 106 recombinant inbred lines as a function of toxin reaction. Disease data are average of four experiments scored on a scale of 1 (resistant) to 5 (susceptible) 7 days postinoculation. Average disease reactions for the parents were 1.0 (Erik) and 4.3 (Kulm). 
gene estimation tool was used to evaluate the hypothesis that loci other than that associated with Ptr ToxA reaction are present and important in the $P$. tritici-repentis race $2-$ wheat interaction. This tool was not used to propose a precise number of genes involved in resistance.

Data reported for the mutants are the average and standard deviation from a single representative experiment. Each experiment involving mutants was repeated and similar results were obtained.

\section{RESULTS}

Toxin reactions. Kulm developed necrosis in response to purified Ptr ToxA $\left(10 \mu \mathrm{g} \mathrm{ml}^{-1}\right)$, but Erik and the mutants (M37, M103, and M322) previously derived from Kulm did not. This is consistent with previously published results (8). Of the $108 \mathrm{RI}$ lines, 54 were homozygous toxin-sensitive, 52 were homozygous toxin-insensitive, and two lines segregated for reaction to $\mathrm{Ptr}$ ToxA. The population is at the $\mathrm{F}_{6}$ generation; therefore, each line can be predicted to be heterozygous at approximately $3.125 \%$ of the loci. The expected segregation ratio for toxin reaction, known to be controlled by a single gene $(7,12)$, was 52.3:52.3:3.4 (toxin sensitive/toxin insensitive/segregating). The observed segregation data in this population fit the expected ratio $\left(\chi^{2}=0.633, P=0.50\right.$ to 0.75 ).

Reaction of RI lines to race 2. The two RI lines that segregated for toxin reaction were not evaluated for reaction to the fungus. Disease reactions for each of the 106 RI lines that were evaluated, averaged across four independent experiments, ranged from 1 to 3.7, with an overall average of 2.0. No transgressive segregation for resistance or susceptibility was observed because average disease ratings on Erik and Kulm were 1.0 and 4.3, respectively. The distribution of disease severities for the entire population was normal but skewed towards resistance (Fig. 1). Only four lines, each toxin sensitive, had disease ratings higher than 3.0 (Fig. 2).

As a group, the 52 toxin-insensitive RI lines tended to be more resistant than the toxin-sensitive lines (Fig. 2). Disease severities for toxin-insensitive lines ranged from 1.0 to 2.6, with an overall average for the group of 1.87 days after inoculation. The highest disease average for a toxin-insensitive line was 2.6; therefore,

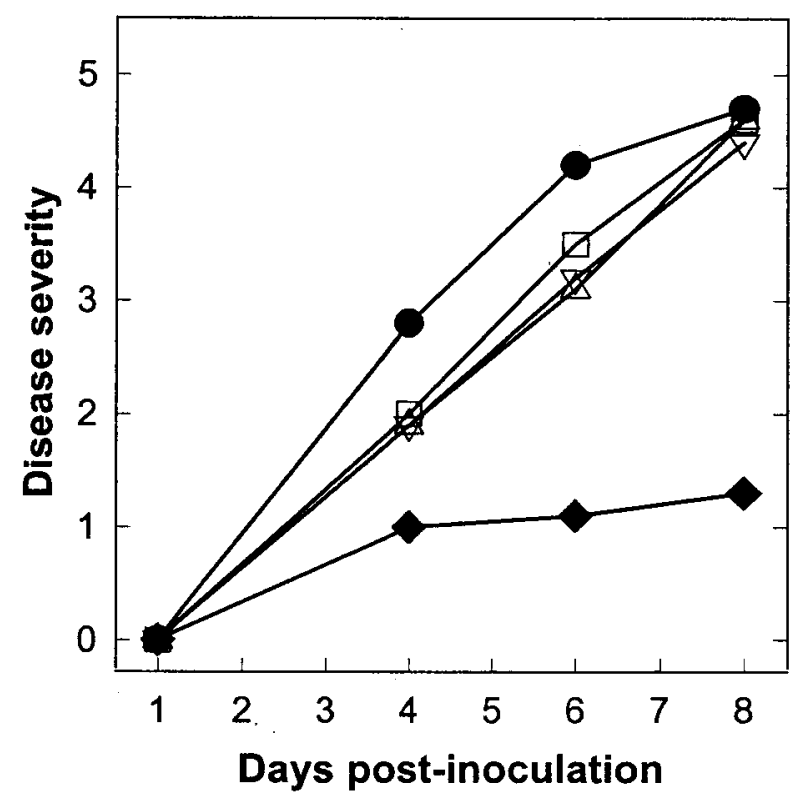

Fig. 3. Time course of disease development for Kulm (•), M37 (O), M103 $(\Delta)$, M322 $(\nabla)$, and Erik $(\diamond)$ inoculated with Pyrenophora tritici-repentis race 2 . none of the 52 insensitive lines was considered susceptible. By comparison, disease severities for each of the 54 toxin-sensitive wheat lines ranged from 1.2 to 3.7 , with an average of 2.3 for the group. Despite sensitivity to Ptr ToxA, 46 of the toxin-sensitive lines were rated as either resistant or moderately resistant to the fungus; 6 of those had disease severity scores $\leq 1.5$ (Fig. 2). Individual lines in the population showed various combinations of toxin and disease reactions, including toxin sensitive-disease resistant, toxin sensitive-disease susceptible, and toxin insensitive-disease resistant (Fig. 2).

The average disease severity for toxin-sensitive lines was $\approx 0.5$ units higher than for the insensitive lines on the 0 -to-5 disease severity scale used. Linear regression analyses indicated that $R^{2}$ between toxin and disease reaction was 0.173 and that toxin
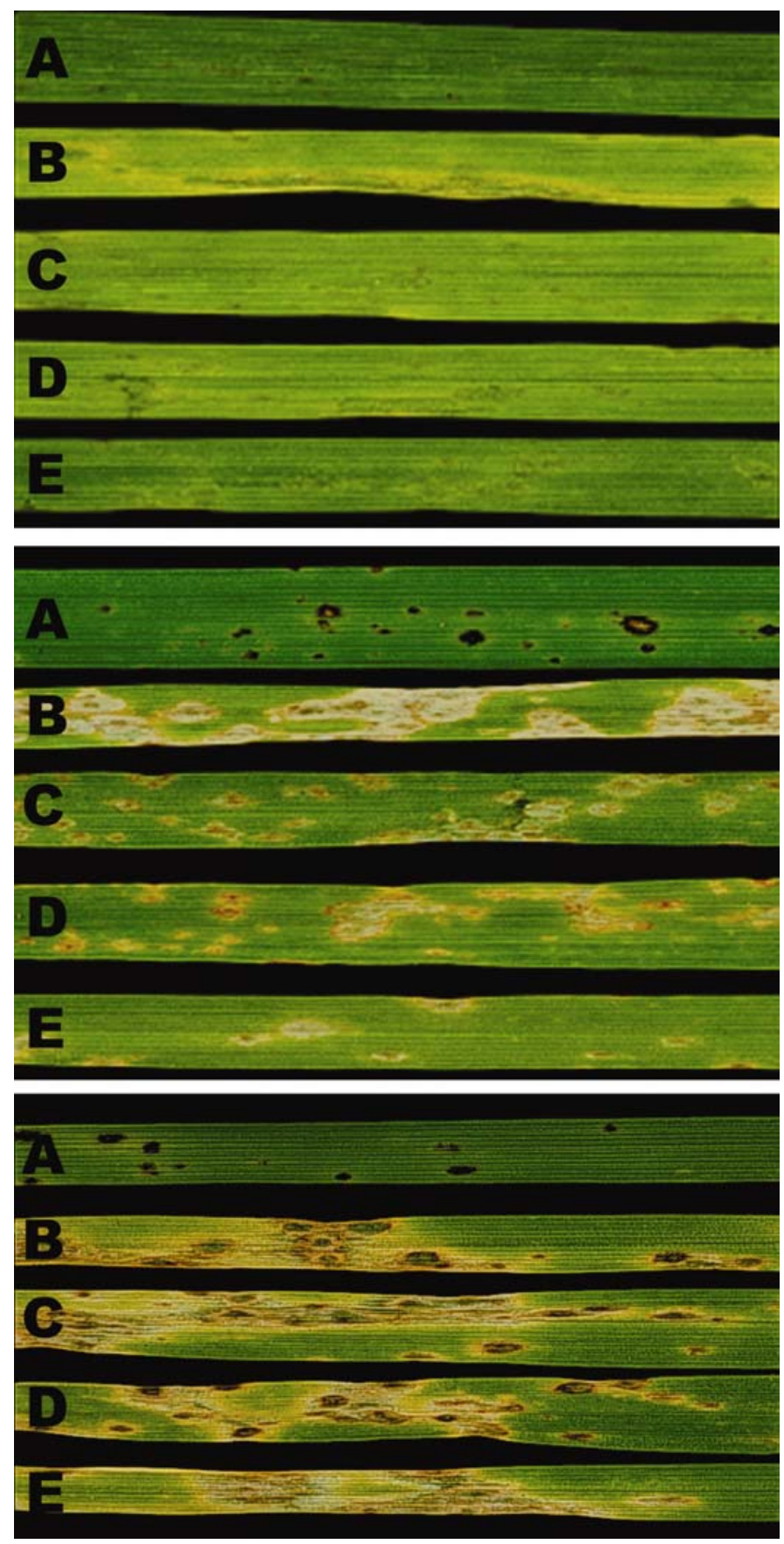

Fig. 4. Reaction of A, Erik, B, Kulm, C, M37, D, M103, and E, M322 to Pyrenophora tritici-repentis race 2 as a function of time. Representative leaves were photographed 3 days (top panel), 5 days (middle panel), and 8 days (bottom panel) postinoculation. 
reaction accounted for $24.4 \%$ of the genetic variance associated with disease. Heritability of resistance was estimated to be 0.714 , and the formula of Falconer and Mackay (6) suggest that between four and five loci in this population were associated with the reaction to $P$. tritici-repentis race 2 .

Reaction of cultivars and mutants to $P$. tritici-repentis race 2. Neither Kulm, Erik, nor any of the mutants showed visible reaction to race 2 at 24- h postinoculation. Accordingly, disease severities for each genotype was 0 at this time point (Fig. 3). By 3 days postinoculation, Kulm wheat showed necrotic lesions, typical of the disease interaction with race 2 (Fig. 4, top panel). Each of the three mutants also had developed necrotic lesions that lacked chlorosis at this time point, but the lesions on each of the mutants were smaller and less severe than those on Kulm. The resistant check Erik showed relatively little in visible response to the fungus 3 days after inoculation, although some small dark brown to black spots typical of resistance were beginning to form (Fig. 4, top panel).

By 5 days postinoculation, the lesions on Kulm and each of the three mutants had increased in size and severity (Fig. 4, middle panel). However, the lesions on Kulm were larger and more severe than those on the three mutants. In addition, there was more coalescence of lesions on Kulm 5 days postinoculation. This resulted in a faster rate of disease development in Kulm than in the mutants in the first 6 days after inoculation. Average disease severity ratings for Kulm were approximately one point higher than those of the three mutants 4 to 6 days postinoculation (Fig. 3).

Each of the three mutants had developed large, severe, coalescing lesions typical of tan spot disease caused by race 2 at 8 days postinoculation. The disease reactions (Fig. 4, lower panel) and average disease severities (Fig. 3) for the mutants were indistinguishable from those on Kulm at this point of the interaction. As expected, Erik was resistant.

Reaction of $\mathbf{F}_{\mathbf{3}}$ families to the fungus. The toxin-insensitive $\mathrm{F}_{3}$ families had average disease severities at 3 days postinoculation that, without exception, were lower than those of the toxin-sensitive $F_{3}$ families (Fig. 5). The six toxin-insensitive families to- gether had an average disease severity of 1.9 at 3 days postinoculation compared with an overall average of 3.1 for the toxinsensitive families at the same time. However, disease severities of each family 8 days postinoculation were 4.0 or higher and, therefore, similar to those on Kulm, regardless of toxin reaction. Overall average disease severities were 4.4 and 4.5 for the toxininsensitive and -sensitive families, respectively, at 8 days postinoculation. Thus, each $\mathrm{F}_{3}$ family ultimately was considered susceptible to race 2 .

\section{DISCUSSION}

The RI population segregated 1:1 for reaction to Ptr ToxA. Toxin sensitivity previously was known to be conditioned by a single dominant gene $(7,12)$; therefore, the data are consistent with an independent assortment of genes in the population. Disease reactions in the population ranged from 1 to 3.7, with average disease reactions for Erik $=1$ and for Kulm $=4.25$. No transgressive segregation was observed for susceptibility, suggesting that Erik lacks susceptibility genes not already present in Kulm. Although no significant resistance genes can be attributed to Kulm in this study, we cannot rule out the possibility that they exist because the average disease score for Erik did not permit detection of transgressive segregants for resistance. Overall, the vast majority of the population was found to possess some level of resistance (disease ratings $\leq 2.9$ ), suggesting the presence of multiple, independent resistance loci or quantitative trait loci in the population. A statistical analysis of the data suggests that between four and five resistance loci may be segregating in this population. This observation is consistent with previous work that concluded that tan spot resistance is controlled by multiple loci in durum and hexaploid wheats $(5,16)$.

RI lines were found that were toxin sensitive-disease susceptible, toxin sensitive-disease resistant, and toxin insensitive-disease resistant. No lines were found in the population that were toxin insensitive and disease susceptible, but only four lines were found to have a level of susceptibility greater than 3.0. Multiple resistance loci may be segregating; therefore, the population of

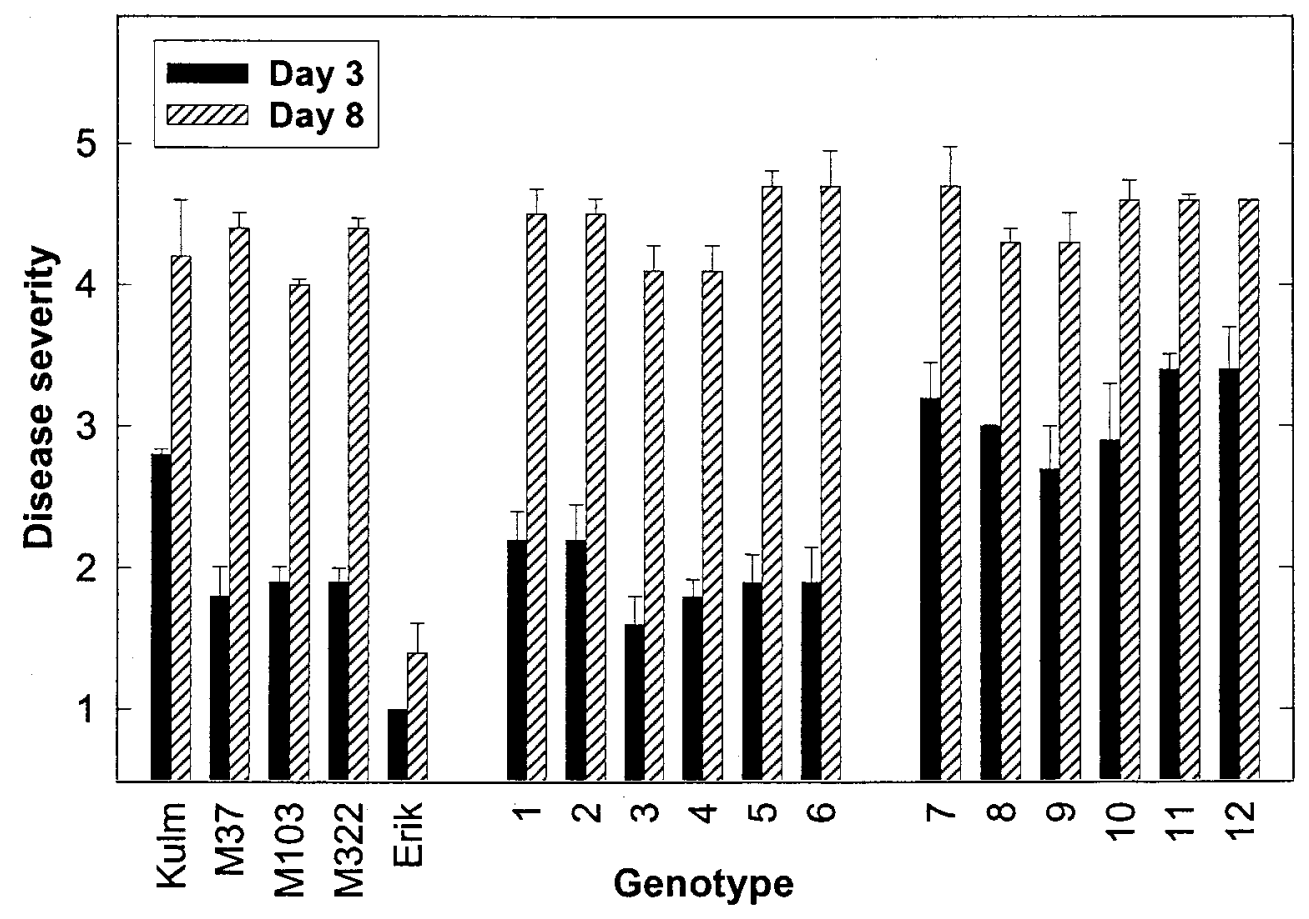

Fig. 5. Average disease severities of homozygous toxin-insensitive (genotypes 1 to 6 ) and homozygous toxin-sensitive (genotypes 7 to 12 ) $F_{3}$ families relative to Kulm, mutants, and Erik at 3 and 8 days postinoculation. Genotypes 1, 2, 7, and 8 were $\mathrm{F}_{3}$ families derived from Kulm $\times$ M37; genotypes 3, 4, 9, and 10 were from Kulm $\times$ M103; and genotypes 5, 6, 11, and 12 were from Kulm $\times$ M322. 
108 lines may have been too small to find a toxin insensitivedisease susceptible line (i.e., a line that is toxin insensitive and lacks other resistance loci). It should be noted, however, that the toxin insensitive-disease susceptible phenotype was demonstrated with the mutants; therefore, all possible toxin-disease reactions in the host were observed in this study.

It was reported previously that toxin insensitivity in the host always was associated with resistance to race 2 (12). That result was not reproducible here, despite the fact that the same race 2 isolate (86-124) was used by both groups. Thus, pathogen variability does not appear to be a reason for the different results. However, different wheat genotypes were used in the two studies. The host populations used in previous studies may have lacked significant resistance (or susceptibility) loci other than that for toxin reaction. If this interpretation is correct, sensitivity to Ptr ToxA may be the key host phenotype for disease susceptibility if other resistance (or susceptibility) loci are not present in the host.

Although the mutants developed tan spot disease at 8 days postinoculation, the lesions developed more slowly on the toxininsensitive genotype than on the wild-type Kulm (Figs. 3 and 4). In previous work, toxin-insensitive wheat mutants were found to be susceptible to $P$. tritici-repentis race 1 (8). In that study, a single disease evaluation was made for each inoculated plant 7 days postinoculation; no effort was made to identify whether disease developed more rapidly on Kulm in response to race 1. It is standard protocol to make a single disease evaluation 6 to 8 days after inoculation in greenhouse tests involving tan spot $(8,10,17,19)$. A more careful evaluation of disease progression that includes evaluations 3 to 6 days postinoculation may reveal that host sensitivity to Ptr ToxA influences the rate of lesion formation in race 1 as well.

In the current study, the lesions that formed on the mutants in response to race 2 possessed a considerable amount of necrosis (Fig. 2). The basis of this is not known. Previous work has demonstrated that the mutants do not respond to toxin concentrations as high as $75 \mu \mathrm{g} \mathrm{ml} \mathrm{l}^{-1}(8)$. Both races 1 and 2 may possess necrosis-inducing metabolites other than Ptr ToxA, though none have been demonstrated unequivocally to date. The toxin-insensitive mutants may be useful in the future for identifying such necrosis-inducing compounds.

Scheffer (18) defined pathogenicity determinants as those molecules required for the establishment of disease. By comparison, virulence factors were defined as pathogen-produced molecules that affected the severity of disease. In the context of these definitions, the data presented here suggest that Ptr ToxA may be a virulence factor rather than a pathogenicity factor for race 2 , at least for some wheat genotypes. Evidence for this includes the observation that toxin sensitivity influenced the rate of lesion formation (Figs. 3 to 5). Also, toxin reaction influenced but did not define the outcome of inoculations in the RI population (Fig. 2), ultimately accounting for $24.4 \%$ of the disease reaction. The data also suggest, but do not directly prove, that race 2 possesses a host range determinant or determinants other than Ptr ToxA. Although it is clear that production of Ptr ToxA is sufficient for pathogenicity on wheat by $P$. tritici-repentis (3), the data reported here raises the question of whether toxin production is necessary for disease on all host genotypes. Elucidating the precise role of Ptr ToxA in tan spot disease will require the isolation and characterization of fungal mutants disrupted at ToxA. Other studies involving the heterologous expression of ToxA in pathogenic fungi that normally do not parasitize wheat also are warranted.

\section{ACKNOWLEDGMENTS}

Financial support was provided by the North Dakota Agricultural Experiment Station. We thank S. Meinhardt for the continuous gifts of purified Ptr ToxA and J. Faris for helpful comments and suggestions concerning the analyses of the RI populations.

\section{LITERATURE CITED}

1. Ali, S., and Francl, L. J. 1998. Race structure of Pyrenophora triticirepentis isolated from wheat and grasses in the U.S. Great Plains. (Abstr.) Phytopathology 88:S114.

2. Ciuffetti, L. M., Francl, L. J., Ballance G. M., Bockus W. W., Lamari, L., Meinhardt, S. W., and Rasmussen, J. B. 1998. Standardization of toxin nomenclature in the Pyrenophora tritici-repentis/wheat interaction. Can. J. Plant Pathol. 20:421-424.

3. Ciuffetti, L. M., Tuori, R. P., and Gaventa, J. M. 1997. A single gene encodes a selective toxin causal to the development of tan spot of wheat. Plant Cell 9:135-144.

4. Effertz, R. J., Meinhardt, S. W., Anderson, J. A., Jordahl, J. G., and Francl, L. J. 2002. Identification of a chlorosis-inducing toxin from Pyrenophora tritici-repentis and the chromosomal location of an insensitivity locus in wheat. Phytopathology 92:527-533.

5. Elias, E, Cantrell, R. G., and Hosford, R. M., Jr. 1989. Heritability of resistance of tan spot in durum wheat and its association with other agronomic traits. Crop. Sci. 29:299-304.

6. Falconer, D. S., and Mackay, F. C. 1996. Pages 208-227 in: Introduction to Quantitative Genetics. Longman Group Ltd., Edinburgh.

7. Faris, J. D., Anderson, J. A., Francl, L. J., and Jordahl, J. G. 1996 Chromosomal location of a gene conditioning insensitivity in wheat to necrosis-inducing culture filtrate from Pyrenophora tritici-repentis. Phytopathology 86:459-463.

8. Friesen, T. L., Rasmussen, J. B., Ali, S., Kwon, C. Y., Francl, L. J., and Meinhardt, S. W. 2002. Reaction of Ptr ToxA-insensitive wheat mutants to Pyrenophora tritici-repentis race 1. Phytopathology 92:38-42.

9. Kwon, C. Y., Rasmussen, J. B., Francl, L. J., and Meinhardt, S. W. 1996. A quantitative bioassay for necrosis toxin from Pyrenophora triticirepentis based on electrolyte leakage. Phytopathology 86:1360-1363.

10. Lamari, L., and Bernier, C. C. 1989. Evaluation of wheat lines and cultivars for reaction to tan spot Pyrenophora tritici-repentis based on lesion size. Can. J. Plant Pathol. 11:49-56.

11. Lamari, L., and Bernier, C. C. 1989. Toxin of Pyrenophora triticirepentis: Host-specificity, significance in disease, and inheritance of host reaction. Phytopathology 79:740-744.

12. Lamari, L., and Bernier, C. C. 1991. Genetics of tan necrosis and extensive chlorosis in tan spot of wheat caused by Pyrenophora triticirepentis. Phytopathology 81:1092-1095.

13. Lamari, L., Gilbert, J., and Tekauz, A. 1998. Race differentiation in Pyrenophora tritici-repentis and survey of physiological variation in western Canada. Can. J. Plant Pathol. 20:396-400.

14. Lamari, L., Sayoud, R., Boulif, M., and Bernier, C. C. 1995. Identification of a new race in Pyrenophora tritici-repentis: Implications for the current pathotype classification system. Can. J. Plant Pathol. 17:312-318.

15. Lander, E. S., Green, P., Abrahomson, J., Barlow, A., Daly, M. J., Lincoln, S. E., and Newburg, L. 1987. MAPMAKER: An interactive computer package for constructing primary genetic linkage maps of experimental and natural populations. Genomics 1:174-181.

16. Nagle, B. J., Frohberg, R. C., and Hosford, R. M., Jr. 1982. Inheritance of resistance to tan spot of wheat. Pages 40-45 in: Tan Spot of Wheat and Related Diseases Workshop. R. M. Hosford, Jr., ed., North Dakota Agricultural Experiment Station, Fargo.

17. Riede, C. R., Francl, L. J., Anderson, J. A., Jordahl, J. G., and Meinhardt, S. W. 1996. Additional sources of resistance to tan spot of wheat. Crop Sci. 36:771-777.

18. Scheffer, R. P. 1983. Toxins as chemical determinants of disease. Pages 1-40 in: Toxins and Plant Pathogenesis. J. M. Daly and B. J. Deverall, eds. Academic Press, Australia.

19. Sykes, E. E., and Bernier, C. C. 1991. Qualitative inheritance of tan spot resistance in hexaploid, tetraploid, and diploid wheat. Can. J. Plant Pathol. 13:38-44.

20. Zhang, H., Francl, L. J., Jordahl, J. G., and Meinhardt, S. W. 1997. Structural and physical properties of a necrosis-inducing toxin from Pyrenophora tritici-repentis. Phytopathology 87:154-160. 